\title{
Dynamic isomiR regulation in Drosophila development
}

\author{
SELENE L. FERNANDEZ-VALVERDE, RYAN J. TAFT, and JOHN S. MATTICK
}

Institute for Molecular Bioscience, The University of Queensland, St Lucia, QLD 4072, Australia

\begin{abstract}
Several recent reports have demonstrated that microRNAs (miRNAs) can exhibit heterogeneous ends and post-transcriptional nontemplate $3^{\prime}$ end additions of uridines or adenosines. Using two small RNA deep-sequencing data sets, we show here that these miRNA isoforms (isomiRs) are differentially expressed across Drosophila melanogaster development and tissues. Specifically, we demonstrate that: (1) nontemplate nucleotide additions of adenosines to miRNA 3' ends are highly abundant in early development; (2) a subset of miRNAs with nontemplate 3' Us are expressed in adult tissues; and (3) the size of at least eight "mature" (unmodified) miRNAs varies in a life-cycle or tissue-specific manner. These results suggest that subtle variability in isomiR expression, which is widely thought to be the result of inexact Dicer processing, is regulated and biologically meaningful. Indeed, a subset of the miRNAs enriched for $3^{\prime}$ adenosine additions during early embryonic development, including miR-282 and miR-312, show enrichment for target sites in developmental genes that are expressed during late embryogenesis, suggesting that nontemplate additions increase miRNA stability or strengthen miRNA:target interactions. This work suggests that isomiR expression is an important aspect of miRNA biology, which warrants further investigation.
\end{abstract}

Keywords: microRNA; GLD-2; adenylation; embryogenesis; miR-282; miR-312; miR-8

\section{INTRODUCTION}

MicroRNAs (miRNAs) are $\sim 19-24$-nucleotide (nt) small RNAs that principally inhibit the translation of partially complementary mRNAs, although a range of other actions, including transcriptional inhibition, have also been recently described (Brodersen and Voinnet 2009). MicroRNA biogenesis begins with transcription of $\mathrm{a} \sim 1-3$-kb primary miRNA transcript, which forms a stem-loop that is recognized and processed by the RNase III Drosha and its partner Pasha (Morlando et al. 2008). The resulting 70-nt pre-miRNA hairpin is transported to the cytoplasm, where it is cleaved by the RNase III Dicer (Dcr-1) and its partner Loquacious (Loqs) (Ghildiyal and Zamore 2009). In Drosophila melanogaster, one strand of the miRNA duplex, known as the "mature miRNA," is then loaded into a member of the Argonaute family of proteins, AGO1. The remaining strand, known as the miRNA*, was long thought to be discarded; however, recent evidence indicates that it can also be loaded into AGO2 (Czech et al. 2009; Ghildiyal et al. 2009; Okamura et al. 2009). Dcr-1 and Loqs-PB (a Loquacious isoform) bind to AGO1 to form the

Reprint requests to: John S. Mattick, Institute for Molecular Bioscience, The University of Queensland, St Lucia, QLD 4072, Australia; e-mail: j.mattick@imb.uq.edu.au; fax: 61-7-3346-2111.

Article published online ahead of print. Article and publication date are at http://www.rnajournal.org/cgi/doi/10.1261/rna.2379610.
RNA-Induced Silencing Complex (RISC) (Marques et al. 2010), which is the primary effector of miRNA action in eukaryotic cells (Davis and Hata 2009).

Several recent reports have shown that in both animals (humans, mice, D. melanogaster, and Caenorhabditis elegans) and plants (Arabidopsis thaliana, Oryza sativa, and Populus trichocarpa) miRNAs exhibit heterogeneous $5^{\prime}$ and $3^{\prime}$ ends, and post-transcriptional nontemplate $3^{\prime}$ end additions of uridines or adenosines (Li et al. 2005; Landgraf et al. 2007; Ruby et al. 2007; Azuma-Mukai et al. 2008; Morin et al. 2008; Reid et al. 2008; Seitz et al. 2008; Ebhardt et al. 2009; Lu et al. 2009). Although the biological relevance of these miRNA isoforms, or isomiRs, has been questioned, there is evidence in A. thaliana that terminal nucleotide additions occur after Dicer processing of the miRNA precursor (Li et al. 2005), and that some variants are differentially loaded into Argonautes (Seitz et al. 2008; Ebhardt et al. 2009). Likewise, in P. trichocarpa, miRNAs that possess adenosine additions are less prone to degradation (Lu et al. 2009).

Two enzymes have been associated with the addition of isomiR nontemplate nucleotides in animals. Zcchc-11 uridylates miR-26a in mouse epithelial cells, leading to the abrogation of the translational repression of human Interleukin 6 (IL-6) (Jones et al. 2009). Similarly, the polynucleotidyl transferase GLD-2 was recently shown to adenylate miR-122 in human and mouse liver (Katoh et al. 2009), which, like adenylation in plants, increases stability. Interestingly, 
the Drosophila homolog of this protein, Wispy, is involved in long-term memory formation (Kwak et al. 2008) and oogenesis (Cui et al. 2008), suggesting that miRNA terminal nucleotide additions may play a role in fly development.

Here we address whether nontemplate additions to miRNAs are biologically regulated, by determining whether such additions show specificity with respect to particular miRNAs and/or developmental stages in Drosophila. Our results show that 3 ' end nontemplate additions change dynamically throughout fly development in a stage- and miRNA-specific manner, and surprisingly, that the size of at least eight miRNAs changes across tissues and development time.

\section{RESULTS}

We analyzed two publicly available $D$. melanogaster small RNAs deep-sequencing data sets generated on both the Roche/ 454 (Ruby et al. 2007) and Illumina (Chung et al. 2008) platforms, the latter of which includes two biological replicates (Supplemental Table S1). We focused our analysis largely on three embryonic development stages ( $0-1 \mathrm{~h}, 2-6 \mathrm{~h}, 6-10 \mathrm{~h}$ ), imaginal discs, and adult heads and bodies. For clarity we will refer to a single deep-sequencing read as a "tag," and the number of times this read appeared in a library as its "count." Of 152 mature miRNAs in miRBase 14.0 we found that 130 were expressed with an absolute expression of 150 counts in at least one time point. The most abundant miRNA species were dominantly $22 \mathrm{nt}$, as expected (Supplemental Fig. S1).

To identify deep-sequencing tags with nontemplate additions we queried for tags that mapped uniquely to known miRNA loci and possessed 1-5 terminal mismatches composed of one or more adenine $(A)$, uracil $(U)$, cytosine $(C)$, or guanine $(\mathrm{G})$ residues at the $3^{\prime}$ end of the tag (Fig. 1A). For simplicity, individual miRNAs or isomiRs with nontemplate $3^{\prime}$ additions are hereafter appended with "- $\mathrm{X}$," where $\mathrm{X}$ is A, U, C, or G (e.g., miR-282-A or isomiR-A).

Although tags with nontemplate additions were more weakly expressed than unmodified tags (e.g., canonical mature miRNAs; Supplemental Figs. S2, S3), those with terminal A (and sometimes $\mathrm{U}$, see below) additions were unexpectedly abundant. We identified 101,173 isomiR-A counts representing $3.9 \%$ of total miRNA expression, and found that $58 \%$ of modified tags were derived from isomiR-As. The majority of
isomiR-As were $23 \mathrm{nt}$ or longer (Supplemental Fig. S1), indicating that in most cases nontemplate nucleotides are added to a "miRNA core" (hereafter denoted miRNA^), which is identical to the mature miRNA (Fig. 1A; Supplemental Fig. S1B), although there are some exceptions and variability in miRNA^ $\wedge$ lengths (see below). This is consistent with a previous report indicating that terminal nucleotides are added after Dicer processing in animals (Katoh et al. 2009).

To test whether A additions occur uniformly or nonrandomly, the percentage of isomiR-As derived from 124 high-confidence miRNAs was calculated. This subset of Drosophila miRNAs is refractory to multimapping (de Hoon et al. 2010), thus ensuring that the observed modifications are not due to spurious matches between highly similar loci. Additionally, to ensure that weakly expressed tags did not bias our analysis, we used a lower threshold of 150 isomiR-A 
counts derived from a single tag per miRNA locus. We observed no correlation between the level of miRNA expression and nontemplate $3^{\prime}$ additions, indicating that isomiR-As are unlikely to be the result of either a random addition in vivo or related to endogenous degradation (Supplemental Fig. S4). Indeed, four highly expressed embryonic miRNAs, including miR-12 and miR-286 (Aravin et al. 2003; Biemar et al. 2005), have $<1 \%$ of their expression driven by isomiR-As (Supplemental Table S2).

IsomiR-A enrichments are consistent between data sets generated on both the Illumina and Roche/454 platforms and are most pronounced early in Drosophila development (Fig. 1B; Supplemental Fig. S5). Indeed, these enrichments are present

in each of the three replicates of each developmental stage or tissue library despite more than eightfold variation in total sequence depth (Supplemental Table S1; Supplemental Fig. S5). To rule out the possibility that the enrichment in isomir-As and isomiR-Us in early Drosophila developmental time points are caused by a systematic deep-sequencing artifact shared by the Illumina and the Roche/454 platforms, we analyzed isomiR expression in three Illumina data sets of 473 human synthetic miRNAs sequenced at equal molarity (Linsen et al. 2009). Consistent with the hypothesis that isomiRs are a biologically meaningful species, we found that in this synthetic RNA set the highest percentage represented by any isomiR variant was $<0.3 \%$ and was randomly distributed between isomiR-As, isomir-Cs, isomiR-Us, and isomiR-Gs, the latter being the most abundant (Supplemental Figs. S6, S7). There was no synthetic miRNA that showed a specific enrichment for isomiR tags (data not shown).

In contrast, when we queried for specific miRNAs enriched for isomiR variants in Drosophila, we identified six miRNAs with a wide range of expression values that were strongly enriched for isomiR-A tags in early development (Supplemental Table S3). Each had $>15 \%$ of its counts driven by a single isomir-A tag in an early embryonic time point (Fig. 2; Supplemental Table S3). For example, in 0-1h embryos miR282-A tags account for $>26 \%$ of miR-282 expression, and overall, constitute nearly one-fourth of all mir- 282 counts in all embryonic time points. Intriguingly, although the dominant miR-282 isoform is $22 \mathrm{nt}$, the most abundant miR-282-A has a miRNA^ $\wedge$ of $21 \mathrm{nt}$ (Fig. 3A), which may suggest that some isomiR-A species result from specific Dicer cleavage events. Similarly, $20 \%$ of miR- 8 expression is driven by a 23-nt isomiR-A in 2-6-h embryos (Fig. 3B), and abundant isomiR-As are derived from both the mature and star strands of miR-312 (Fig. 3C).

We also identified a further nine miRNAs with 5\%-15\% of their total counts driven by isomiR-As and found that, like the highly modified miRNA discussed above, all show peak isomiR-A abundance in early development (Supplemental Fig. S8). This strongly suggests that nontemplate A additions are linked with fly development. Indeed, isomiR-A expression amongst all 15 modified miRNAs is highly replicable between the early embryonic libraries in both the Roche/454 and Illumina data sets (Supplemental Fig. S8), and is strongly diminished or undetectable in adult biological replicates and samples derived from larval and pupal life-cycle stages (Supplemental Fig. S9).

MicroRNAs with similar expression profiles can act cooperatively to target a subset of genes or a particular molecular pathway (Enright et al. 2003; Bartel 2009). To test whether similar patterns were present amongst the six miRNAs with highly abundant isomiR-As, we queried TargetScanFly 5.7 (Ruby et al. 2007) and identified 33 genes with two target sites, and a further five genes with three target sites. Using FlyMine (Lyne et al. 2007) we found that these 38 genes are significantly enriched for eight Gene Ontology (GO) terms (Table 1), most of which refer to developmental and morphogenic processes, including instar, larval, and pupal development. A similar GO term analysis on our less-stringent isomiR-A set, comprised of 15 miRNAs (see above), revealed 118 genes with two or more target sites that show a similar but more extensive GO enrichment (Supplemental Table S4). At least $68 \%$ of GO terms in this set correspond to biological processes that take place in late development. The observation that isomiR-As are more abundant during early development, and the fact that their target genes are active in developmental processes suggests that isomiR-As delay or stabilize miRNA action.

We also found 46,821 counts from isomiR-U tags, comprising nearly $2 \%$ of total miRNA expression and $27 \%$ of all modified isomiR counts. While nontemplate $U$ additions are less common than As, and only four miRNAs have $>150$ isomiR-U counts and $>5 \%$ of their expression constituted by these tags, we found that the former are moderately enriched 


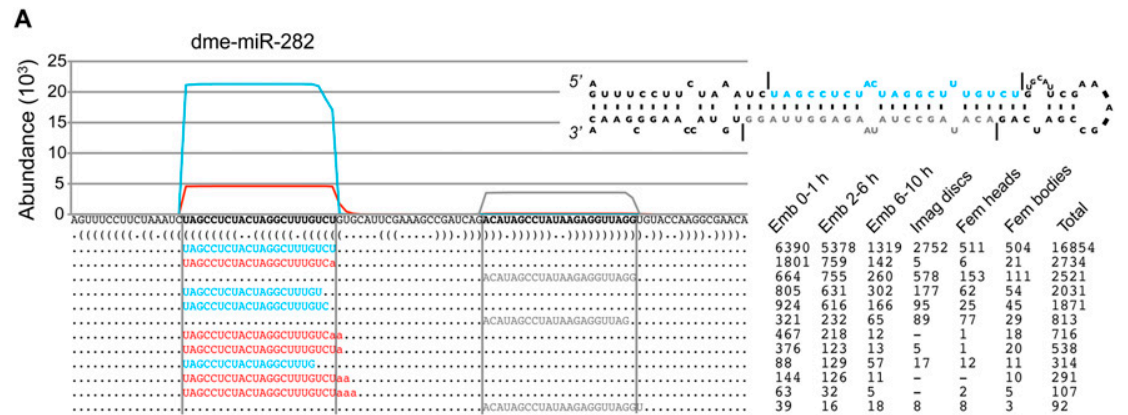

B

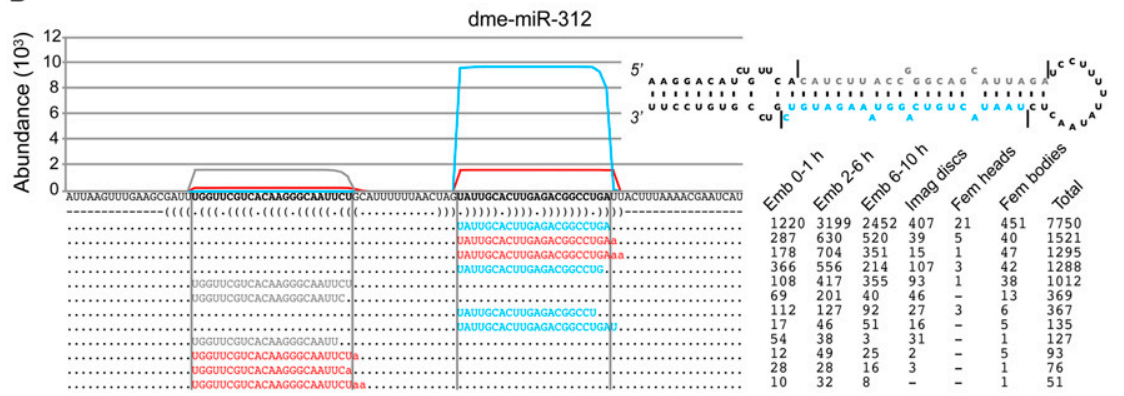

C

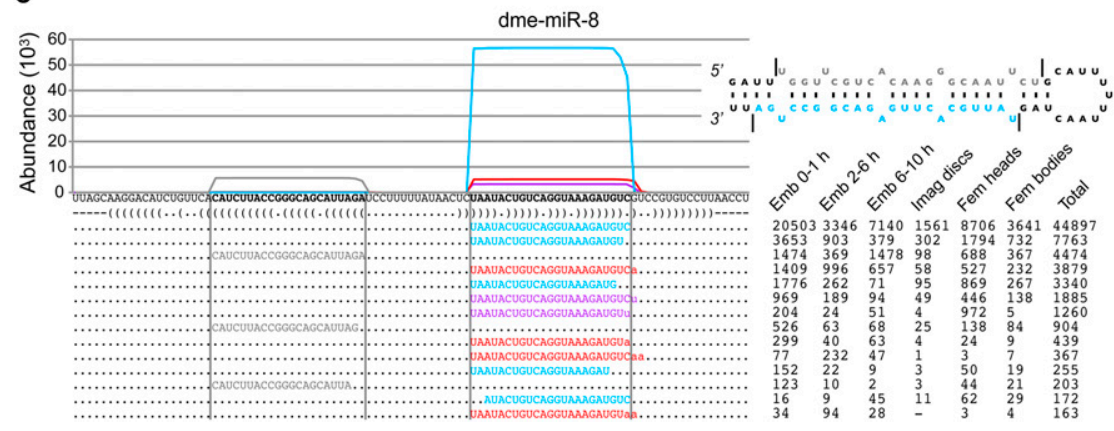

FIGURE 3. IsomiRs derived from dme-mir-282, dme-mir-312, and dme-mir-8. The most abundant unmodified and isomiR tags derived from dme-mir-282 $(A)$, dme-mir-312 $(B)$, and dme-mir- $8(C)$ are shown. Unmodified tags are shown in blue, isomiR-A tags are shown in red, isomiR-U tags are shown in purple, and miRNA* tags are shown in gray. The counts for each tag are given on the right. The terms embryo, imaginal, and female are abbreviated as "Emb," "Imag," and "Fem," respectively, in the column headers. Tag counts were retrieved form the deepest replicate of the GSE11624 data set.

in adult tissues (Supplemental Fig. S10). Interestingly, we found that miR- 8 shows evidence of developmental regulation of both $3^{\prime} \mathrm{U}$ and A additions. While the dominant miR-8-A tags from 2-6-h embryos (20\% of expression, 1232 counts) has a miRNA^ $\wedge$ of $23 \mathrm{nt}$, the dominant miR-8-U in female heads ( $8 \%$ of expression, 1042 counts) has a miRNA^ of 22 nt, further suggesting that terminal nucleotide additions are regulated post-Dicer events (Figs. 2, 3C; Fig. S10).

Despite the fact that miRNA $5^{\prime}$ and $3^{\prime}$ end variation is well documented, it is frequently assumed that the dominant isoform (i.e., the mature miRNA) expressed from any given locus is constant across time and tissue type, and that variation in expression is restricted to weakly expressed (and presumably aberrant) isomiRs. To investigate whether mature miRNA sizes are, in fact, invariable, we examined the size of the most abundant unmodified tags derived from each of the
124 high-confidence miRNAs across developmental time and anatomical region. Surprisingly, we identified eight cases where the dominant isoform changes dramatically (Fig. 4A); in some cases, by as much as 10 -fold (Fig. 4B). These isomiRs share the same $5^{\prime}$ start sequence, but shift in length. For example, the primary isoform expressed by miR-124 is $22 \mathrm{nt}$ in Drosophila embryos, but 22- and 21-nt species are equally abundant in imaginal discs, and adult bodies and heads almost exclusively produce 21-nt species (Fig. 4B). Likewise, miR-12 shows a shift from 20-nt species in 0-1-h embryos to 23-nt species in 6-10-h embryos, and equivalent production of both in adult heads and bodies (Fig. 4A). These results suggest that variations in mature miRNA size are regulated events.

\section{DISCUSSION}

Here, we have shown (1) that isomiRs with nontemplate $3^{\prime}$ As are specifically enriched in early developmental stages amongst miRNAs that target genes active in development, (2) that a small subset of miRNAs with nontemplate $3^{\prime}$ Us are expressed in adult tissues, and (3) that the size of the mature miRNA can change across developmental time or anatomic region.

Similar to adenylation of mRNAs (Barnard et al. 2004), it is possible that isomiR-As act to regulate maternally inherited miRNAs and ensure the success of the embryonic developmental program by making them resistant to degradation and/or altering their temporal activity. Indeed, miR-282, 26\% of whose embryonic expression is driven by isomiR-As, has been genetically linked to dorso-ventral boundary establishment in Drosophila embryos (Bejarano et al. 2008). Indeed, stabilization of miRNAs by adenylation has been previously shown in black cottonwood (Lu et al. 2009) and mouse (Katoh et al. 2009). Additionally, a recent study has shown segmentspecific repression by another highly modified miRNA, miR312, in Drosophila embryos (Reich et al. 2009), suggesting that adenosine additions may stabilize miRNAs in certain regions, while facilitating their degradation in others. In fact, both functions have been previously ascribed to mRNA poly(A) tails and are essential for successful Drosophila embryo development (Benoit et al. 2008).

A GLD family ("defective in Germ Line Development") protein is the likely Drosophila miRNA adenosyltransferase 
TABLE 1. Target site Gene Ontology enrichment for miRNAs with abundant isomiR-As

\begin{tabular}{lccc}
\hline GO ID & GO description & Number of genes $\left(\%^{*}\right)$ & $P$-value \\
\hline GO:0007435 & Salivary gland morphogenesis & $4(19)$ & $4.28 \times 10^{-4}$ \\
GO:0022612 & Gland morphogenesis & $4(19)$ & $4.28 \times 10^{-4}$ \\
GO:0007431 & Salivary gland development & $4(19)$ & $7.59 \times 10^{-4}$ \\
GO:0035272 & Exocrine system development & $4(19)$ & $7.59 \times 10^{-4}$ \\
GO:0048732 & Gland development & $4(19)$ & $1.27 \times 10^{-3}$ \\
GO:0009653 & Anatomical structure morphogenesis & $9(43)$ & $1.93 \times 10^{-3}$ \\
GO:0002165 & Instar larval or pupal development & $5(24)$ & $3.61 \times 10^{-3}$ \\
GO:0009791 & Post-embryonic development & $5(24)$ & $4.39 \times 10^{-3}$ \\
GO:0009888 & Tissue development & $5(24)$ & $6.62 \times 10^{-3}$ \\
GO:0045449 & Regulation of transcription & $6(29)$ & $7.17 \times 10^{-3}$ \\
\hline
\end{tabular}

(*) Percentage calculated over the 21 genes that have Gene Ontology term annotation. naute complexes), facilitate localization to subcellular regions, or regulate target choice or the intensity of miRNA action. Overall, the precise and replicable alterations in miRNA size, which appear to be dependent on both temporal and anatomic cues, suggest that variations in miRNA expression might be analogous to mRNA alternative splicing.

The fact that we find evidence for adenylation and reproducible generation of different isomiR variants from several single genomic miRNA locus points to the possibility that similar strategies regulate the presence of both mRNAs and candidate. The cytoplasmic poly(A) polymerase Wispy (GLD2 in other animals) is responsible for adenosine additions to miR-122 in humans and mice, and has been linked to oocyte maturation in mice, Xenopus laevis, C. elegans, M. musculus, and Drosophila (Kadyk and Kimble 1998; Barnard et al. 2004; Nakanishi et al. 2006; Benoit et al. 2008; Cui et al. 2008). Maternal Drosophila wispy mutants show defects in bicoid mRNA localization and microtubule activity in female meiosis, leading to early developmental arrest (Brent et al. 2000). bicoid expression is activated by cytoplasmic polyadenylation in early embryogenesis (Coll et al. 2010). Consistent with our finding that isomiR-A levels are most abundant early in development, high levels of Wispy have been detected in adult female bodies, oocytes, and 0-2-h embryos (Cui et al. 2008). It is possible that Wispy adenylates miRNAs during oogenesis and/or early in Drosophila development.

It is widely held that, other than the "mature miRNA" (defined in miRBase), the wide diversity of small RNAs derived from any given miRNA locus are nonfunctional byproducts of inexact of pre-miRNA cleavage. We have shown that the size of at least eight mature miRNAs changes in a developmental stage or tissue-specific manner. Although we prefer the hypothesis that these species are the result of regulated Dicer processing events, our data cannot resolve whether this is indeed the case or whether they are the result of degradation or $3^{\prime}$ exonuclease activity. Irrespective of their biogenesis, however, their precise and highly reproducible expression in two different deep experiments carried out in two different platforms suggests they are biologically regulated, thus likely to be functional. Indeed, size variant isomiRs share a common 5 ' end, and therefore also a common "seed" sequence (nucleotides $2-8$ of the mature miRNA), which guides mRNA targeting (Farazi et al. 2008). For example, two members of the miR-9 family show "mature switching," but possess exactly the same seed sequence and differ only in their $3^{\prime}$ end (Fig. 4A). This may suggest that miRNA $3^{\prime}$ end binding or targeting is more important than previously thought, as has been reproposed recently (Lee et al. 2009). Indeed, small changes to miRNA $3^{\prime}$ ends may facilitate or inhibit loading of these variants onto Argonaute proteins (or alternative Argo-
miRNAs during development. Further studies should reveal whether embryonic expression of isomiR-As is shared amongst metazoans, as well as to elucidate the mechanisms by which sequencing technologies combined with the simultaneous identification of miRNA and isomiR targets may soon shed light on their biological functions.

\section{MATERIALS AND METHODS}

\section{Data set selection and mapping strategy}

We analyzed two publicly available small RNA deep-sequencing data sets (18-26 nt with $5^{\prime}$ monophosphate and $\left.3^{\prime} \mathrm{OH}\right)$ from the Gene Expression Omnibus Database (GEO) (http://www.ncbi.nlm. nih.gov/geo/). Data series GSE11624 was sequenced using the Illumina platform (Chung et al. 2008), while data series GSE7448 was sequenced using the Roche/454 platform (Ruby et al. 2007). These data sets were selected due to their depth and breadth of coverage, and the availability of unmapped (i.e., raw or adaptor trimmed) sequences. Three raw runs of a set of 473 synthetic miRNAs sequenced in the Illumina platform were obtained from GEO series GSE16374 (Linsen et al. 2009).

Each data set was retrieved and the adaptor sequence was trimmed, if necessary, using a custom-generated script (http:// www.biopieces.org). The resulting sequence tags were mapped to the D. melanogaster genome (UCSC dm3, BDGP Release 5) using Bowtie (Langmead et al. 2009). Tags that could not be mapped with 0 mismatches were trimmed $1 \mathrm{nt}$ from their $3^{\prime}$ end and mapped again to the genome; this step was iterated until up to 5 nt were clipped from the $3^{\prime}$ end of the unmappable tags. The synthetic miRNA tags were mapped using the same strategy, but against the Homo sapiens genome (UCSC hg18, NCBI Build 36.1).

Only tags that mapped uniquely to the sense strand of premiRNA in miRBase (miRBase Release 14.0, http://microrna.sanger. ac.uk/sequences/index.shtml) (Griffiths-Jones et al. 2008) were analyzed further.

\section{Analysis of deep-sequencing data}

Only tags that mapped perfectly to the mature/star miRNA locus (as reported in miRBase) and tags that had one or more mononucleotide stretches in their $3^{\prime}$ end that did not correspond to the isomiR production is regulated. In addition, advances in deep- 
A

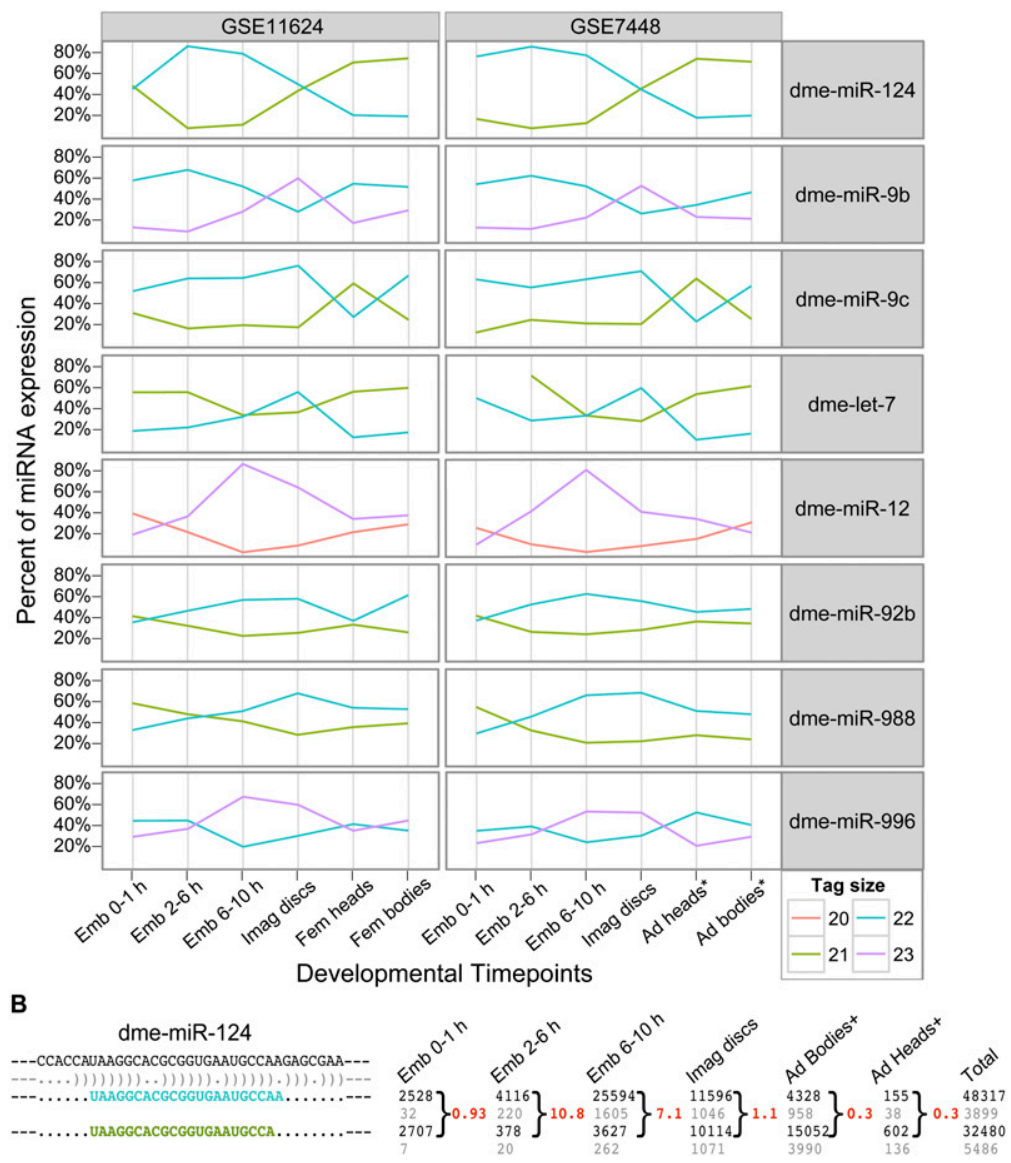

FIGURE 4. Differential isomiR expression. (A) The expression of the two most abundant unmodified tags for each miRNA is shown. These tags share the same $5^{\prime}$ end and differ only in length at their $3^{\prime}$ end. The size of each tag is differentiated by color-20 nt (pink), $21 \mathrm{nt}$ (green), $22 \mathrm{nt}$ (blue), $23 \mathrm{nt}$ (purple). Data are shown for the deepest of the GSE11624 replicate samples and GSE7448. (B) The two most abundant dme-miR-124 tags are shown in blue and green and correspond to the 21- and 22-nt species depicted in the bottom of $A$. The table on the right displays the count for each tag in the deepest GSE11624 replicate (black) and GSE7448 (gray). The terms embryo, imaginal, female, and adult are abbreviated as "Emb," "Imag," "Fem," and "Ad," respectively, in the column headers in $B$ and the $x$-axis of $A$. The ratio of abundance (blue/green) for the GSE11624 samples is highlighted in red beside column. $\left(^{*}\right)$ Samples obtained from male and female tissues. $(+)$ Counts from female (black) and male and female tissues (gray).

genomic sequence were retrieved and considered as modified isomiRs.

To ensure that the modifications we observed were transcribed from a specific miRNA locus and not due to incorrect matching of a tag produced by a miRNA locus of another member of the same miRNA family, we analyzed 124 miRNAs with exclusively singlemapping isomiR tags. We only regarded a miRNA as modified if it had at least 150 isomiR-A or isomir- $U$ counts and constituted $>5 \%$ of total miRNA expression. For each of these miRNAs, the counts of each isomiR tag were recorded and the percentage that isomiR type represented in each sample was calculated to correct for sequencing depth. To compare each individual miRNA locus across samples, we recalculated the percentage of total expression each tag represented over the total counts of modified and unmodified isomiR tags derived from each miRNA loci.
These analyses were performed using a series of perl scripts (available upon request) and the R statistical analysis software. Plotting was performed with the $\mathrm{R}$ package ggplot2 (http://had.co.nz/ggplot2/).

\section{Gene ontology enrichment analysis}

We selected the miRNAs that had $>15 \%$ isomiR-A counts driven by a single tag in at least one time point (shown in Fig. 2) and identified the genes that had two or more target sites for those miRNAs according to TargetScanFly 5.1 (Ruby et al. 2007). This yielded 38 genes, which were then queried for Gene Ontology Enrichments using FlyMine (http://www.flymine.org/) (Lyne et al. 2007). We only retained GO terms enriched with $P$-values higher than 0.01 (after Bonferroni correction) and at least four genes per term. A similar procedure was followed for 118 genes that were targeted by miRNAs that had $>150$ isomir-A counts representing at least 5\% of their expression (Supplemental Fig. S8), just changing the enrichment threshold to 0.001 and more than five genes per enrichment.

\section{Isomir identification}

Altered size isomiRs were identified by retrieving the two most abundant isoforms in each developmental time point from the deepest GSE11624 replicate (marked with an asterisk $\left.{ }^{*}\right]$ in Supplemental Table S1) and the GSE7448 data set. Only one replicate of GSE11624 was analyzed, because there are no replicates for the imaginal disc sample in this data set. We focused on miRNA loci with $>150$ counts of altered size isomiR tags, the most abundant of which represented $>20 \%$ of that miRNA expression. We then asked whether the most abundant isomiR was the same in all developmental time points, and found eight examples (Fig. 4), in which two isomiRs changed in relative abundance in both data sets. These analyses were also performed using a series of perl scripts (available upon request) and the $\mathrm{R}$ statistical analysis software.

\section{DATA SUBMISSION}

Data used in this analysis is publicly available at NCBI's Gene Expression Omnibus (http://www.ncbi.nlm.nih.gov/geo/) with Accession Series numbers GSE11624, GSE7448, and GSE16374.

\section{SUPPLEMENTAL MATERIAL}

Supplemental material can be found at http://www.rnajournal.org. 


\section{ACKNOWLEDGMENTS}

We thank Dr. Martin Hansen for access to and assistance with the Biopieces toolset (http://code.google.com/p/biopieces/). We also thank Sam Linsen for helping us access the raw sequencing reads of the synthetic miRNA deep sequencing data. J.S.M., R.J.T., and S.L.F.V. are supported by a Federation Fellowship grant (FF0561986) and a Discovery Project grant (DP0988851) from the Australian Research Council.

Received February 5, 2010; accepted July 21, 2010.

\section{REFERENCES}

Aravin AA, Lagos-Quintana M, Yalcin A, Zavolan M, Marks D, Snyder B, Gaasterland T, Meyer J, Tuschl T. 2003. The small RNA profile during Drosophila melanogaster development. Dev Cell 5: 337-350.

Azuma-Mukai A, Oguri H, Mituyama T, Qian ZR, Asai K, Siomi H, Siomi MC. 2008. Characterization of endogenous human Argonautes and their miRNA partners in RNA silencing. Proc Natl Acad Sci 105: 7964-7969.

Barnard DC, Ryan K, Manley JL, Richter JD. 2004. Symplekin and xGLD-2 are required for CPEB-mediated cytoplasmic polyadenylation. Cell 119: 641-651.

Bartel DP. 2009. MicroRNAs: Target recognition and regulatory functions. Cell 136: 215-233.

Bejarano F, Luque CM, Herranz H, Sorrosal G, Rafel N, Pham TT, Milán M. 2008. A gain-of-function suppressor screen for genes involved in dorsal-ventral boundary formation in the Drosophila wing. Genetics 178: 307-323.

Benoit P, Papin C, Kwak JE, Wickens M, Simonelig M. 2008. PAPand GLD-2-type poly(A) polymerases are required sequentially in cytoplasmic polyadenylation and oogenesis in Drosophila. Development 135: 1969-1979.

Biemar F, Zinzen R, Ronshaugen M, Sementchenko V, Manak JR, Levine MS. 2005. Spatial regulation of microRNA gene expression in the Drosophila embryo. Proc Natl Acad Sci 102: 15907-15911.

Brent AE, MacQueen A, Hazelrigg T. 2000. The Drosophila wispy gene is required for RNA localization and other microtubule-based events of meiosis and early embryogenesis. Genetics 154: 16491662.

Brodersen P, Voinnet O. 2009. Revisiting the principles of microRNA target recognition and mode of action. Nat Rev Mol Cell Biol 10: 141-148.

Chung W-J, Okamura K, Martin R, Lai EC. 2008. Endogenous RNA interference provides a somatic defense against Drosophila transposons. Curr Biol 18: 795-802.

Coll O, Villalba A, Bussotti G, Notredame C, Gebauer F. 2010. A novel, noncanonical mechanism of cytoplasmic polyadenylation operates in Drosophila embryogenesis. Genes Dev 24: 129-134.

Cui J, Sackton KL, Horner VL, Kumar KE, Wolfner MF. 2008. Wispy, the Drosophila homolog of GLD-2, is required during oogenesis and egg activation. Genetics 178: 2017-2029.

Czech B, Zhou R, Erlich Y, Brennecke J, Binari R, Villalta C, Gordon A, Perrimon N, Hannon GJ. 2009. Hierarchical rules for Argonaute loading in Drosophila. Mol Cell 36: 445-456.

Davis BN, Hata A. 2009. Regulation of microRNA biogenesis: A miRiad of mechanisms. Cell Commun Signal 7: 18. doi: 10.1186/ 1478-811X-7-18.

de Hoon MJL, Taft RJ, Hashimoto T, Kanamori-Katayama M, Kawaji H, Kawano M, Kishima M, Lassmann T, Faulkner GJ, Mattick JS, et al. 2010. Cross-mapping and the identification of editing sites in mature microRNAs in high-throughput sequencing libraries. Genome Res 20: 257-264.

Ebhardt HA, Tsang HH, Dai DC, Liu Y, Bostan B, Fahlman RP. 2009. Meta-analysis of small RNA-sequencing errors reveals ubiquitous post-transcriptional RNA modifications. Nucleic Acids Res 37: 2461-2470.

Enright AJ, John B, Gaul U, Tuschl T, Sander C, Marks DS. 2003. MicroRNA targets in Drosophila. Genome Biol 5: R1. doi: 10.1186/ gb-2009-10-2-r25.

Farazi TA, Juranek SA, Tuschl T. 2008. The growing catalog of small RNAs and their association with distinct Argonaute/Piwi family members. Development 135: 1201-1214.

Ghildiyal M, Zamore PD. 2009. Small silencing RNAs: An expanding universe. Nat Rev Genet 10: 94-108.

Ghildiyal M, Xu J, Seitz H, Weng Z, Zamore P. 2009. Sorting of Drosophila small silencing RNAs partitions microRNA* strands into the RNA interference pathway. RNA 16: 43-56.

Griffiths-Jones S, Saini HK, Van Dongen S, Enright AJ. 2008. miRBase: Tools for microRNA genomics. Nucleic Acids Res 36: D154-D158.

Jones MR, Quinton LJ, Blahna MT, Neilson JR, Fu S, Ivanov AR, Wolf DA, Mizgerd JP. 2009. Zcchc11-dependent uridylation of microRNA directs cytokine expression. Nat Cell Biol 11: 11571163.

Kadyk LC, Kimble J. 1998. Genetic regulation of entry into meiosis in Caenorhabditis elegans. Development 125: 1803-1813.

Katoh T, Sakaguchi Y, Miyauchi K, Suzuki T, Kashiwabara S-I, Baba T, Suzuki T. 2009. Selective stabilization of mammalian microRNAs by $3^{\prime}$ adenylation mediated by the cytoplasmic poly(A) polymerase GLD-2. Genes Dev 23: 433-438.

Kwak JE, Drier E, Barbee SA, Ramaswami M, Yin JCP, Wickens M. 2008. GLD2 poly(A) polymerase is required for long-term memory. Proc Natl Acad Sci 105: 14644-14649.

Landgraf P, Rusu M, Sheridan R, Sewer A, Iovino N, Aravin AA, Pfeffer S, Rice A, Kamphorst AO, Landthaler M, et al. 2007. A mammalian microRNA expression atlas based on small RNA library sequencing. Cell 129: 1401-1414.

Langmead B, Trapnell C, Pop M, Salzberg S. 2009. Ultrafast and memory-efficient alignment of short DNA sequences to the human genome. Genome Biol 10: R25. doi: 10.1186/gb-2009-10$3-\mathrm{r} 25$.

Lee I, Ajay SS, Yook JI, Kim HS, Hong SH, Kim NH, Dhanasekaran SM, Chinnaiyan AM, Athey BD. 2009. New class of microRNA targets containing simultaneous $5^{\prime}$-UTR and $3^{\prime}$-UTR interaction sites. Genome Res 19: 1175-1183.

Li J, Yang Z, Yu B, Liu J, Chen X. 2005. Methylation protects miRNAs and siRNAs from a 3'-end uridylation activity in Arabidopsis. Curr Biol 15: 1501-1507.

Linsen SEV, de Wit E, Janssens G, Heater S, Chapman L, Parkin RK, Fritz B, Wyman SK, de Bruijn E, Voest EE, et al. 2009. Limitations and possibilities of small RNA digital gene expression profiling. Nat Methods 6: 474-476.

Lu S, Sun Y-H, Chiang VL. 2009. Adenylation of plant miRNAs. Nucleic Acids Res 37: 1878-1885.

Lyne R, Smith R, Rutherford K, Wakeling M, Varley A, Guillier F, Janssens H, Ji W, Mclaren P, North P, et al. 2007. FlyMine: An integrated database for Drosophila and Anopheles genomics. Genome Biol 8: R129. doi: 10.1186/gb-2007-8-7-r129.

Marques JT, Kim K, Wu P-H, Alleyne TM, Jafari N, Carthew RW. 2010. Loqs and R2D2 act sequentially in the siRNA pathway in Drosophila. Nat Struct Mol Biol 17: 24-30.

Morin RD, O'Connor MD, Griffith M, Kuchenbauer F, Delaney A, Prabhu AL, Zhao Y, McDonald H, Zeng T, Hirst M, et al. 2008. Application of massively parallel sequencing to microRNA profiling and discovery in human embryonic stem cells. Genome Res 18: 610-621.

Morlando M, Ballarino M, Gromak N, Pagano F, Bozzoni I, Proudfoot NJ. 2008. Primary microRNA transcripts are processed cotranscriptionally. Nat Struct Mol Biol 15: 902-909.

Nakanishi T, Kubota H, Ishibashi N, Kumagai S, Watanabe H, Yamashita M, Kashiwabara S-I, Miyado K, Baba T. 2006. Possible role of mouse poly(A) polymerase mGLD-2 during oocyte maturation. Dev Biol 289: 115-126. 
Okamura K, Liu N, Lai EC. 2009. Distinct mechanisms for microRNA strand selection by Drosophila Argonautes. Mol Cell 36: 431-444.

Reich J, Snee MJ, Macdonald PM. 2009. miRNA-dependent translational repression in the Drosophila ovary. PLoS ONE 4: e4669. doi: 10.1371/journal.pone.0004669.

Reid JG, Nagaraja AK, Lynn FC, Drabek RB, Muzny DM, Shaw CA, Weiss MK, Naghavi AO, Khan M, Zhu H, et al. 2008. Mouse let-7 miRNA populations exhibit RNA editing that is constrained in the $5^{\prime}$-seed/cleavage/anchor regions and stabilize predicted mmu-let7a:mRNA duplexes. Genome Res 18: 1571-1581.

Ruby JG, Stark A, Johnston WK, Kellis M, Bartel DP, Lai EC. 2007. Evolution, biogenesis, expression, and target predictions of a substantially expanded set of Drosophila microRNAs. Genome Res 17: 1850-1864.

Seitz H, Ghildiyal M, Zamore PD. 2008. Argonaute loading improves the $5^{\prime}$ precision of both microRNAs and their miRNA strands in flies. Curr Biol 18: 147-151. 

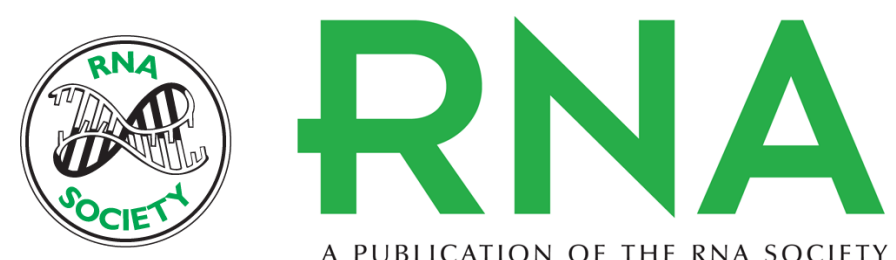

A PUBLICATION OF THE RNA SOCIETY

\section{Dynamic isomiR regulation in Drosophila development}

Selene L. Fernandez-Valverde, Ryan J. Taft and John S. Mattick

RNA 2010 16: 1881-1888 originally published online August 30, 2010

Access the most recent version at doi:10.1261/rna.2379610

Supplemental

Material

References This article cites 41 articles, 13 of which can be accessed free at:

http://rnajournal.cshlp.org/content/16/10/1881.full.html\#ref-list-1

\section{License}

Email Alerting

Service

http://rnajournal.cshlp.org/content/suppl/2010/08/11/rna.2379610.DC1

top right corner of the article or click here. 